\title{
Optimization and Validation of a High-Performance Liquid Chromatography Method for the Analysis of Hesperidin and Carvacrol for Veterinary Use
}

\author{
Eleonora Truzzi ${ }^{1}$, Stefania Benvenuti1 ${ }^{*}$, Davide Bertelli ${ }^{1}$, Maurizio Scozzoli ${ }^{2}$ \\ ${ }^{1}$ Department of Life Sciences, University of Modena and Reggio Emilia, Modena, Italy \\ ${ }^{2}$ APA-CT S.R.L., Forlì, Italy \\ Email: *stefania.benvenuti@unimore.it
}

How to cite this paper: Truzzi, E., Benvenuti, S., Bertelli, D. and Scozzoli, M. (2021) Optimization and Validation of a High-Performance Liquid Chromatography Method for the Analysis of Hesperidin and Carvacrol for Veterinary Use. American Journal of Analytical Chemistry, 12, 459-470.

https://doi.org/10.4236/ajac.2021.1212029

Received: October 19, 2021

Accepted: December 10, 2021

Published: December 13, 2021

Copyright $\odot 2021$ by author(s) and Scientific Research Publishing Inc. This work is licensed under the Creative Commons Attribution International License (CC BY 4.0).

http://creativecommons.org/licenses/by/4.0/

\begin{abstract}
Plant-derived compounds have been recognized by the feed industry as important supplements for livestock welfare and health. In this context, Citrus aurantium L. extract and Origanum vulgare L. essential oil have been demonstrated to have strong anti-inflammatory and antioxidant effects on animals. Being the composition of plant-derived extracts extremely influenced by the environmental and growing conditions of the plants, quality control is necessary in terms of the concentration of the active compounds to assure the reproducibility of natural feed additives. The present work aimed at the validation of the extraction procedure from feed additives of Hesperidin (HES) and Carvacrol (CAR), the main active compounds of Citrus aurantium and Origanum vulgare extracts. Then, the quantification method of both the analytes was developed and validated by reversed high-performance liquid chromatography coupled with a UV detector. The validated method was tested on premixtures and final feed additives supplied by a local feed factory to supervise the production chain. The extraction method with methanol resulted to be efficient and highly reproducible, with recovery higher than $90 \%$ for both the analytes. The chromatographic method has been demonstrated to be accurate, precise (relative standard deviation percent lower than $2.06 \%$ ), and linear in the tested range concentrations, with regression coefficients equal to 0.995 and 0.999 for HES and CAR respectively. The method demonstrated that the feed additives prepared by the factory by diluting the premixtures were less concentrated than what was declared on the label.
\end{abstract}

\section{Keywords}

Origanum vulgare, Citrus aurantium, Quality Control, Feed Additives 


\section{Introduction}

Intensive animal agriculture incredibly increased in the last decades due to a higher demand of animal-derived food (such as milk and meat). Intensive farm practices are more convenient for profit, more efficient for the management, and ensure affordable food prices. However, intensive farming has seriously compromised animal life and welfare by confinement [1]. As a consequence of livestock management, animals are affected by chronic stress, inflammatory and infectious diseases which might seriously impact animal health and performance [2] [3]. Therefore, the employment of synthetic non-steroidal anti-inflammatory drugs and antibiotics is a common practice in farms for disease treatment. In addition to these, other veterinary drugs are used for treatment, such as tranquilizers, antiparasitic, anthiasmatics, growth promoters, or for prophylaxis of inflammatory diseases [4] [5]. However, their administration is essential for livestock health and productivity, the widespread use of these substances represents a risk for consumers [6] [7]. Indeed, recent reports have highlighted the presence of drug residues in animal-derived products, such as milk, eggs, and meat [8] [9] [10]. In recent years, strong market pressure is being applied on livestock to become Clean, Green, and Ethical (CGE). The CGE approach involves the reduction of synthetic drugs, the reduction of the impact of animal industries on the environment, and the improvement of animal welfare [11]. Furthermore, in 2006, the European Union banned the employment of antibiotic growth promoters in animal nutrition to fight the emergence of antibiotic-resistant bacteria [12]. In this context, Phytogenic Feed Additives (PFAs) represent a valid alternative to the use of synthetic drugs due to the antioxidant, antimicrobial, and anti-inflammatory activities of plant bioactive compounds [13] [14] [15]. In addition to these therapeutic properties, they demonstrated to ameliorate the performance and the quality of animal products by acting as immunomodulators and digestive stimulants [16] [17]. PFAs are commonly classified as various secondary plant metabolites (phenolic acids, terpenes, flavonoids, and essential oils), and they might be recovered from plant food industry by-products in the perspective of a circular economy, with the aim to reduce industrial waste and increasing environmental sustainability [18]. Moreover, the focus on employing "recycled" secondary plant metabolites in livestock highlights the possibility of their usage in organic farms [19].

In the last years, the dietary effects of Citrus extract (Citrus aurantium L.) and oregano essential oil (Origanum vulgare L.) have been demonstrated on different animal-producing food, and their employment is admitted as feed additives [12]. Hesperidin (HES) is the most important flavanone glycoside found in the peel of citrus fruits, and it is normally recovered from the wastes of the food industry [20] [21] [22]. HES has shown good anti-inflammatory and antioxidant properties in animals, and therefore has the potential to prevent chronic diseases [23]. Furthermore, several authors recently proved that HES supplementation could ameliorate the composition, antioxidant, and microbial qualities of chick- 
en meat [24] [25] [26] [27] [28]. Oregano essential oil is extracted by steam distillation and it is commonly used in the food industry and feed production as a flavoring agent. The beneficial activities of oregano essential oil are mainly attributed to carvacrol and thymol which are the most abundant monoterpenes. Carvacrol (CAR) and thymol are phenolic monoterpenes with a strong free-radical scavenging action, conferring the essential oil antioxidant and anti-inflammatory properties [29]. In addition, CAR has been demonstrated to exert a beneficial probiotic effect in porcine, and positive effects on intestinal morphology in poultry [30] [31].

The administration of citrus extract and oregano essential oil, along with other natural extracts, as feed additives in livestock nutrition, could represent a valid solution to enhance animal welfare. The quality control of feed additives or their premixtures (PMs) is necessary to assure the reproducibility of the beneficial effects. In addition, also the raw materials employed for the production of PMs should be characterized regarding the concentration of active compounds to establish the dilution for the production of the final formulation. Thus, the present work aimed at the development of an efficient extraction procedure of HES and CAR from both PMs and final feed additives for their quantification. Furthermore, a reverse-phase High-Performance Liquid Chromatography (HPLC) UV method was validated for the simultaneous quantification of the analytes. The chemical structures of HES and CAR are displayed in Figure 1.

\section{Method}

\subsection{Materials}

Two PMs and three final feed supplements (FS) were kindly gifted by APA-CT S.r.l. (Forli, Italy). The PMs were obtained with a powder blender containing

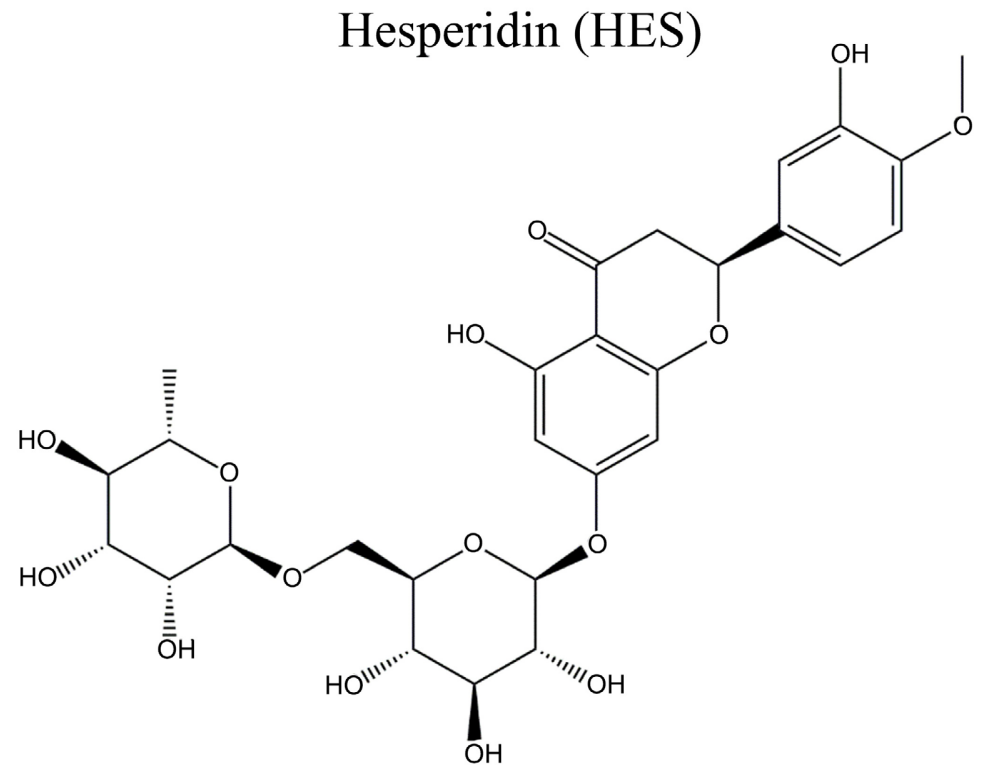

Carvacrol (CAR)<smiles>Cc1ccc(C(C)C)cc1O</smiles>

Figure 1. Chemical structures of Hesperidin (HES) and Carvacrol (CAR). 
hydroalcoholic extracts of Echinacea spp., Rosa canina, Glycyrrhiza glabra, Citrus aurantium, and essential oil of Origanum vulgare. The excipients contained in the PMs were inorganic materials. The supplied FS are dilutions of the PMs at different concentrations: FS-A1 and FS-A2 were obtained from PM-A at the final concentration of $40 \%$ and $10 \% \mathrm{w} / \mathrm{w}$ of the PM in silica, respectively. Finally, FS-B1 was obtained by diluting PM-B with dextrose at the concentration of $10 \%$ $\mathrm{w} / \mathrm{w}$.

For the HPLC method validation, HES and CAR were provided from Extrasynthese (Genay, France) and Sigma-Aldrich (Milan, Italy) respectively. The Internal Standard (IS) methyl parahydroxybenzoate was purchased from Carlo Erba Reagenti (Milan, Italy).

Acetonitrile (ACN), methanol ( $\mathrm{MeOH})$, dimethyl sulfoxide (DMSO) were provided from Sigma-Aldrich (Milan, Italy). All the solvents were of analytical grade.

\subsection{Preparation of Standard Solutions}

Stock standard solutions of HES $(0.2 \mathrm{mg} / \mathrm{mL})$ and CAR $(2 \mathrm{mg} / \mathrm{ml})$ were prepared by dissolving them in $\mathrm{MeOH}$. The Internal Standard (IS) solution was prepared in $\mathrm{MeOH}$ at the concentration of $1.65 \mathrm{mg} / \mathrm{mL}$. All solutions were stored protected from light at $4^{\circ} \mathrm{C}$ until use. Under these conditions, the drugs and the IS resulted to be stable up to one month.

\subsection{Extraction of HEP and CARV from PMs and FSs}

The samples were extracted by dynamic maceration. Briefly, a weighed amount of the sample (2.5 g of the PMs, and $5 \mathrm{~g}$ of the FSs) was extracted with $25 \mathrm{~mL}$ of $\mathrm{MeOH}$ under magnetic stirring for $4 \mathrm{~h}$. The solution was then filtered under reduced pressure. The residue was extracted with the same procedure for a second time with $15 \mathrm{~mL}$ of $\mathrm{MeOH}$ for $30 \mathrm{~min}$, and for a third time with $10 \mathrm{~mL}$ of $\mathrm{MeOH}$ for $30 \mathrm{~min}$. The filtrates were combined and adjusted at the final volume of 50 $\mathrm{mL}$ with $\mathrm{MeOH}$ in a volumetric flask. Before the addition of the extracting solvent, $2.5 \mathrm{~mL}$ of IS solution were added to obtain in the final solution the concentration of $82.75 \mu \mathrm{g} / \mathrm{mL}$.

To evaluate the efficiency of the extraction, the solid-to-solvent ratio was decreased. Thus, the procedure was also conducted by extracting $1 \mathrm{~g}$ of the PMs (the samples with the highest content of active ingredients) with the same procedure described above.

\subsection{Chromatographic Conditions}

The HPLC analyses were performed on an Agilent 1100 system (Agilent Technologies, Waldbronn, Germany) equipped with a quaternary pump, an autosampler, and a UV detector. System management and data acquisition were carried out by the HP ChemStation software. Chromatographic separation was achieved with an Ascentis express C18 column $(15 \mathrm{~cm} \times 3.0 \mathrm{~mm} \times 2.7 \mu \mathrm{m})$ (Supelco, 
PA, USA) at $25^{\circ} \mathrm{C}$. The mobile phase consisted of $\mathrm{H}_{2} \mathrm{O}$ (A) and ACN (B). The gradient elution was set as follows: 0 to $5 \mathrm{~min}$, isocratic at $20 \%$ (B); $5-20 \mathrm{~min}$, linear gradient from $20 \%$ to $80 \%$ (B); $20-22 \mathrm{~min}$, isocratic at $80 \%$ (B). The equilibration period was $10 \mathrm{~min}$ long and the total run time was $32 \mathrm{~min}$. The flow rate was set at $0.4 \mathrm{~mL} / \mathrm{min}$ and the injection volume was $3 \mu \mathrm{L}$. The detection wavelength was $278 \mathrm{~nm}$.

\subsection{Method Validation}

The present study was validated in agreement with the international guidelines for analytical methods for quality control of veterinarian products (VICH guidelines) [32]. The validation of HPLC-UV method was based on the following criteria: linearity, precision, accuracy, Limit of Detection (LOD), Limit of Quantitation (LOQ), and recovery.

1) Calibration and quality control samples

Ten-point calibration curves were used to determine linearity. Calibration samples of HES and CAR were prepared in the range of $2.00-90.00 \mu \mathrm{g} / \mathrm{mL}$ and $5.00-170.00 \mu \mathrm{g} / \mathrm{mL}$ respectively, by diluting the standard stock solutions. The internal standard was added to all the working solutions at the final concentration of $82.75 \mu \mathrm{g} / \mathrm{mL}$. All calibration curves were analyzed in triplicate. Linearity was assessed by plotting the ratio between drug peak areas and IS peak area versus the corresponding drug concentrations. Least-squares linear regression fits were performed by using GraphPad Prism 8.4.3 (GraphPad Software, Inc., San Diego, CA).

Quality Control (QC) samples were prepared at three different concentration levels of analytes. The concentrations were $2.00,45.00$, and $90.00 \mu \mathrm{g} / \mathrm{mL}$ for HES, and $5.00,100.00$, and $170.00 \mu \mathrm{g} / \mathrm{mL}$ for CAR.

2) Precision and accuracy

The precision and the accuracy of the chromatographic method (intra- and inter-day variation) were evaluated by replicated analyses of the QC samples at low, medium, and high levels ( $\mathrm{n}=5$, each). Method precision was expressed as percent relative standard deviations (RDS \%) of the "found" values: RSD\% = $(\mathrm{SD} / \mathrm{Mean}) \times 100$. Method accuracy was obtained by comparing the concentration obtained from the calibration curve with the true concentration value of the analytes. Results are expressed as a percent of the estimated concentration.

3) LOD and LOQ

Limit of Detection (LOD) and Limit of Quantitation (LOQ) were calculated as signal-to-noise ratio 3:1 and 10:1 respectively, for each analyte. The LOD and the LOQ concentrations were validated by analyzing drug solutions at the same concentrations.

4) Recovery

The recovery of the extraction was assessed by comparing the level of HES and CAR in the extracted samples (spiked before extraction) to that of unextracted samples (at low, medium, and high concentration levels $(\mathrm{n}=3$, each). The excipients used for feed additives formulation were mixed at the same ratio as the real sam- 
ples and used as matrix for the recovery.

\section{Results and Discussion}

The feed industry has recognized plant-derived compounds as important supplementary additives for livestock in recent years. Indeed, PFAs include both flavoring and bioactive ingredients, essentials for animal welfare and growth. Plantderived extracts may largely vary in composition depending on the environmental conditions or might degrade during feed preparation processes. As a consequence, for both the feed industry and the farmers it is important to quantify the concentration of the beneficial ingredients. In this manner, the feed ration can be optimized depending on the livestock and the final positive effect of nutrients can be controlled and reproducible [33]. In the present work, the validated method for the simultaneous quantification of HES and CAR was applied on a defective product batch of final feed additives prepared starting from concentrated PMs.

\subsection{Chromatography}

The chromatographic conditions were selected focusing on the development of a simple and fast method preserving the chromatographic resolution of the target analytes. The selected column and the mobile phase consisting of acetonitrile and water resulted suitable for the chromatographic separation of HES and CAR. The linear gradient from $20 \%$ to $80 \%$ between 5 and 20 min was set to reduce the elution time of CAR. In this way, the chromatographic run lasted $22.000 \mathrm{~min}$ in total. Methyl parahydroxybenzoate was selected as IS for a similar structure to CAR and its scarce presence in nature. Indeed, being the feed additives prepared with plant extracts, other compounds similar to the analytes (such as flavonoids and phenolic acids) might be present. Moreover, the IS showed an intermediate retention time with respect to the analytes, without interfering with their retention windows (Figure 2). In particular, HES was quickly eluted after $6.830 \mathrm{~min}$, IS at 8.696, and CAR at 18.200 .

The repeatability of the retention time of the analytes was satisfactory, with $\mathrm{RSD} \%$ values lower than $0.7 \%$ for all the analytes. The repeatability was assessed on 45 individual analyses of the QC samples at three different levels (Table 1).

\subsection{Method Validation}

Regarding the validation of the analytical method, the selectivity could not be assessed, since it was not possible to analyze the blank matrix composed by the ethanolic extracts of different plants and excipients. For both HES and CAR the linearity was adequate in the tested range with correlation coefficient values higher than 0.990 (Table 2).

The intra- and inter-day precision, accuracy, and recovery were evaluated at three different levels and the results are displayed in Table 3. The precision values were satisfactory, being in all cases lower than $10 \%$ at the three different 


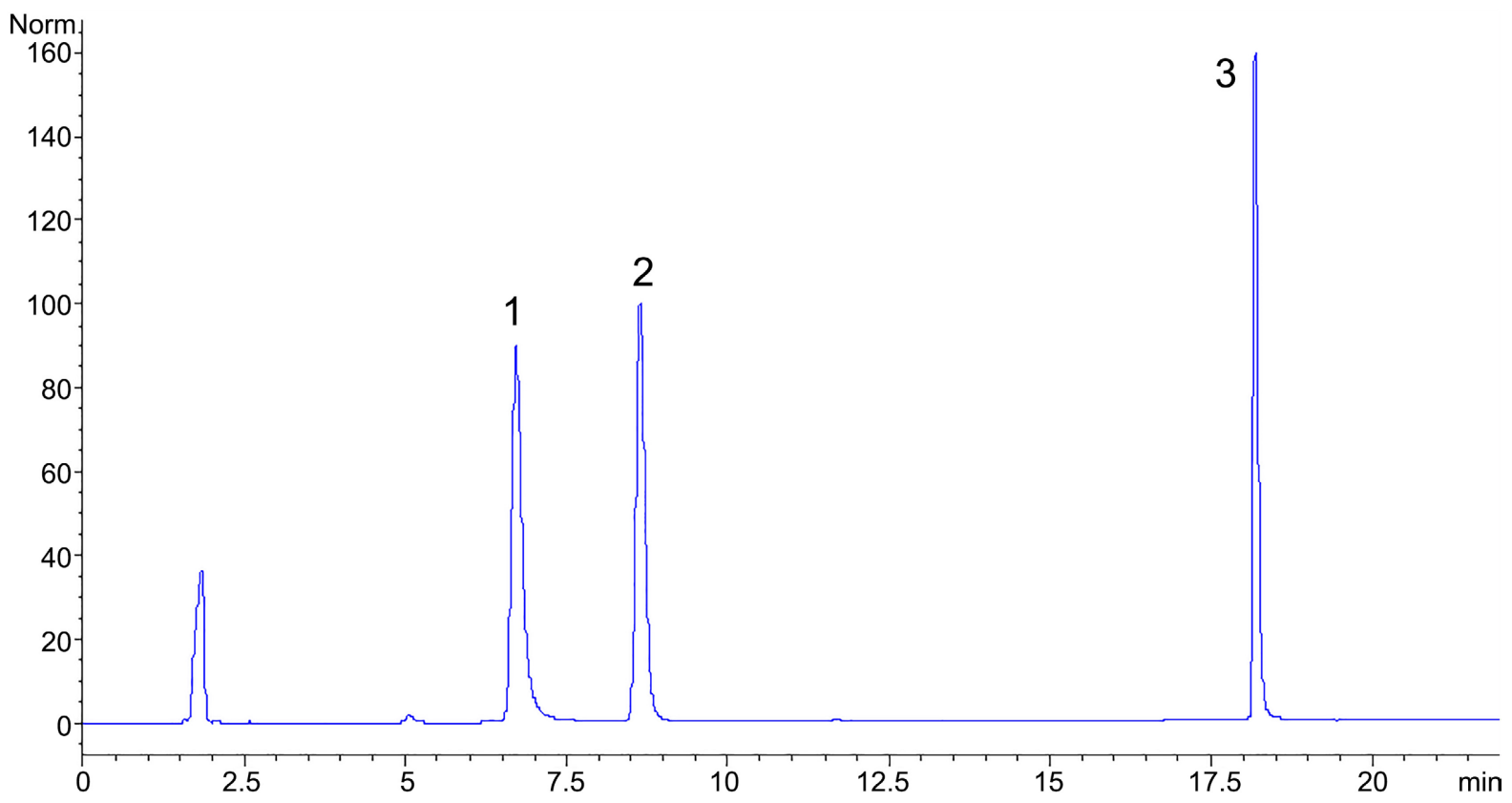

Figure 2. Representative chromatogram of a calibration sample containing $90.00 \mu \mathrm{g} / \mathrm{mL}$ of hesperidin $(1), 82.75 \mu \mathrm{g} / \mathrm{mL} \mathrm{of}$ IS (2) and $170.00 \mu \mathrm{g} / \mathrm{mL}$ of carvacrol (3).

Table 1. Relative Standard Deviation \% (RSD \%) of the retention times of Hesperidin (HES), Carvacrol (CAR), and Internal Standard (IS) on different days at three different concentration levels $(2.00,45.00$, and $90.00 \mu \mathrm{g} / \mathrm{mL}$ for HES, and 5.00, 100.00, and $170.00 \mu \mathrm{g} / \mathrm{mL}$ for CAR).

\begin{tabular}{|c|c|c|c|c|c|c|c|c|c|c|c|c|}
\hline \multirow{2}{*}{ Drug } & \multicolumn{3}{|c|}{ Day 1} & \multicolumn{3}{|c|}{ Day 2} & \multicolumn{3}{|c|}{ Day 3} & \multicolumn{3}{|c|}{ Inter-Day } \\
\hline & $\mathrm{L}$ & M & $\mathrm{H}$ & $\mathrm{L}$ & M & $\mathrm{H}$ & $\mathrm{L}$ & M & $\mathrm{H}$ & $\mathrm{L}$ & M & $\mathrm{H}$ \\
\hline HES & 0.46 & 0.17 & 0.48 & 0.42 & 0.63 & 0.61 & 0.12 & 0.12 & 0.22 & 0.44 & 0.38 & 0.60 \\
\hline CAR & 0.03 & 0.01 & 0.07 & 0.05 & 0.06 & 0.04 & 0.02 & 0.02 & 0.00 & 0.04 & 0.06 & 0.06 \\
\hline IS & 0.44 & 0.11 & 0.40 & 0.44 & 0.32 & 0.36 & 0.21 & 0.15 & 0.08 & 0.44 & 0.42 & 0.60 \\
\hline
\end{tabular}

Note: L, low; M, medium; H, high.

Table 2. Calibration curve parameters (number of points equal to 30), LOD and LOQ values of Hesperidin (HES), and Carvacrol (CAR).

\begin{tabular}{ccccccc}
\hline Drug & Linearity Range & Slope $( \pm$ SE $)$ & Intercept $( \pm$ SE $)$ & R2 & LOD $(\mu \mathrm{g} / \mathrm{mL})$ & $\mathrm{LOQ}(\mu \mathrm{g} / \mathrm{mL})$ \\
\hline HES & $2.00-90.00 \mathrm{ug} / \mathrm{mL}$ & $0.01149( \pm 0.0002)$ & $0.007751( \pm 0.0002)$ & 0.995 & 0.30 & 0.97 \\
CAR & $5.00-170.00 \mathrm{ug} / \mathrm{mL}$ & $0.00517( \pm 0.00003)$ & $+0.00469( \pm 0.00469)$ & 0.999 & 0.23 & 0.76 \\
\hline
\end{tabular}

Table 3. Method validation data: precision (intra- and inter-day), accuracy, and recovery values at low, medium, and high concentrations $(2.00,45.00$, and $80.00 \mu \mathrm{g} / \mathrm{mL}$ for HES, and 5.00, 100.00 , and $170.00 \mu \mathrm{g} / \mathrm{mL}$ for CAR) in the quality control samples.

\begin{tabular}{|c|c|c|c|c|c|c|c|c|c|c|c|c|}
\hline \multirow{2}{*}{ Drug } & \multicolumn{6}{|c|}{ Intra-day precision (RSD \%) Inter-day precision (RSD \%) } & \multicolumn{3}{|c|}{ Accuracy \% } & \multicolumn{3}{|c|}{ Recovery \% } \\
\hline & $\mathrm{L}$ & M & $\mathrm{H}$ & $\mathrm{L}$ & M & $\mathrm{H}$ & $\mathrm{L}$ & M & $\mathrm{H}$ & $\mathrm{L}$ & M & $\mathrm{H}$ \\
\hline HES & 0.71 & 0.27 & 0.37 & 1.00 & 1.01 & 2.06 & 100.5 & 102.7 & 100.9 & 95.81 & 89.99 & 91.03 \\
\hline CAR & 0.51 & 0.18 & 0.95 & 1.36 & 0.64 & 1.74 & 97.84 & 96.8 & 100.25 & 100.26 & 96.86 & 90.05 \\
\hline
\end{tabular}

Note: L, low; M, medium; H, high. 
concentration levels. For this reason, the proposed method can be considered as precise for all analytes. Regarding the method accuracy, the values were within the accepted limits in the range of $90 \%-110 \%$.

Regarding the recovery, all the values at the three concentration levels were higher than or equal to $90 \%$, suggesting that the extraction method was suitable for both HES and CAR at all tested concentrations.

\subsection{Development of Extraction Procedure}

Being HES and CAR both lipophilic compounds, the extraction from the feed additives was attempted by using different organic solvents, namely DMSO, $\mathrm{MeOH}$, and isopropanol. Among these, $\mathrm{MeOH}$ demonstrated to be the most suitable for the analytes and the IS. Several authors argued that $\mathrm{MeOH}$ is the best extraction solvent for HES from citrus peel, and the extraction time and solid-to-solvent ratio were fundamental parameters [20] [34] [35]. The same evidence was observed in our experiments. Indeed, the highest yield for HES was reached by decreasing the solid-to-solvent ratio to 1:25 ratio and increasing the extraction time to $5 \mathrm{~h}$. In particular, it was noticed that increasing yields were obtained by protracting the first extraction step up to $4 \mathrm{~h}$, where about $90 \%$ of HES was extracted. Mauricio et al. reported that hot $\mathrm{MeOH}$ markedly reduced the extraction time of HES from orange albedo [34]. In our case, the same strategy could not be applied due to CAR high volatility. The effectiveness of the optimized extraction procedure of HES and CAR from the PMs was demonstrated by decreasing the solid-to-solvent ratio (1g extracted with a total of $50 \mathrm{~mL}$ of $\mathrm{MeOH}$ ). No differences in the yields were observed, suggesting that the extraction procedure was exhaustive. Thus, all the samples were extracted in triplicate to determine the concentration of the analytes in PM-A and PM-B, and their diluted FSs. Typical chromatograms of the samples and the quantitative results are displayed in Figure 3 and Table 4 respectively.

As can be observed in Figure 3, in the extracts of the feed additives, HES and

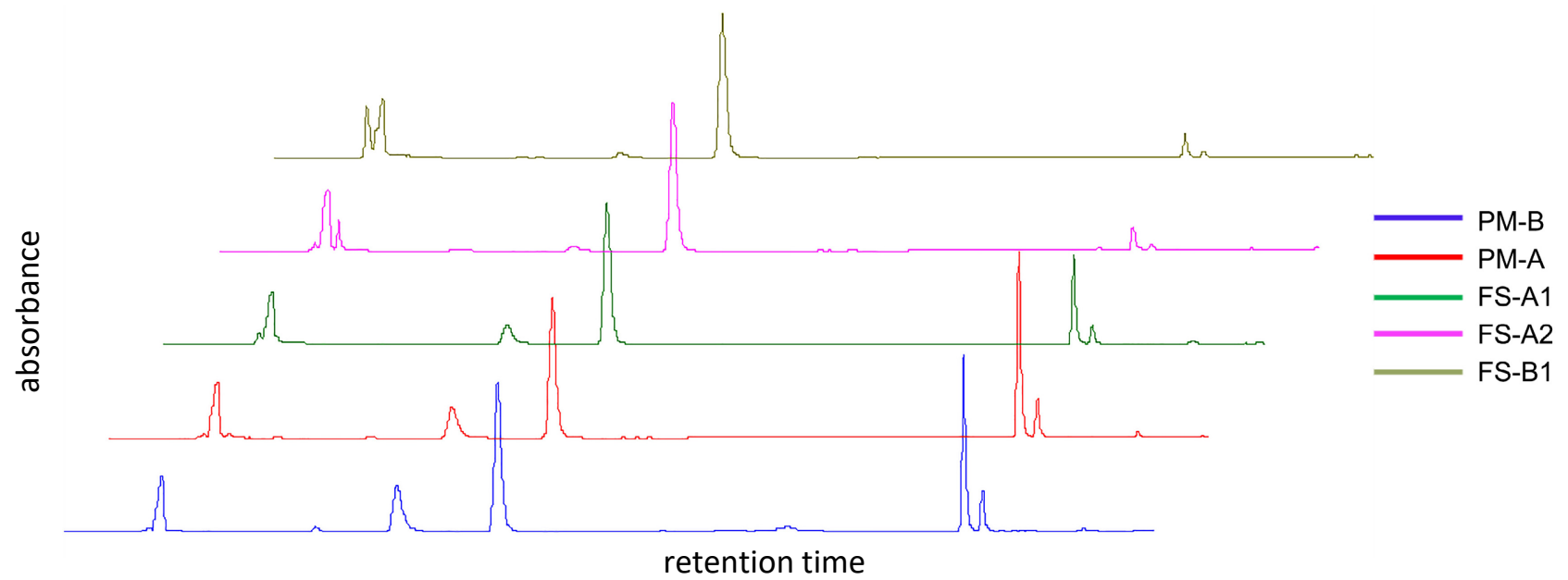

Figure 3. Typical chromatograms of the feed additives PM-A, PM-B, FS-A1, FS-A2, FS-B1. 
Table 4. Quantitative results of HES and CAR extracted from the samples. The results are expressed as mean \pm standard deviation of three replicates. FS samples were prepared by the manufacturer by diluting PM-A and PM-B with dextrose or colloidal silica.

\begin{tabular}{ccccc}
\hline Sample & $\begin{array}{c}\text { Dilution } \\
\text { declared in label }\end{array}$ & HES $(\mathrm{mg} / \mathrm{g})$ & CAR $(\mathrm{mg} / \mathrm{g})$ & Dilution found \\
\hline PM-A & - & $0.597 \pm 0.029$ & $2.747 \pm 0.064$ & - \\
PM-B & - & $0.785 \pm 0.013$ & $2.379 \pm 0.051$ & - \\
& & & & HES: $29.75 \%$ \\
FS-A1 & 40\% PM-A & $0.178 \pm 0.010$ & $0.652 \pm 0.049$ & CAR: $23.72 \%$ \\
& & & & HES: $9.17 \%$ \\
FS-A2 & 10\% PM-A & $0.055 \pm 0.002$ & $0.136 \pm 0.007$ & CAR: $4.96 \%$ \\
& & & & HES: $5.86 \%$ \\
FS-B1 & $10 \%$ PM-B & $0.046 \pm 0.001$ & $0.138 \pm 0.003$ & CAR: $5.78 \%$ \\
\hline
\end{tabular}

CAR were the main compounds present. The peak at 18.547 was identified as thymol, the structural isomer of CAR highly abundant in the essential oil of oregano [36].

The FS resulted in less concentration compared to what was stated on labels. In particular, CAR amounts in FS samples were lower than HES. This evidence might be explained by CAR loss during the mixing procedure with the diluting excipients, due to its high volatility. In order to assure that the lower content of the analytes was due to errors during the manufacturing process, home-made diluted samples with the same excipients were extracted and analyzed. The recovery for HES and CAR ranged from $89 \%$ to $105 \%$ and $88 \%$ to $96 \%$ respectively, confirming that the diluting excipients or the extraction procedure did not significantly affect the extraction of the analytes.

\section{Conclusion}

The quality control of final or intermediate products involved in the production chain of feed additives is extremely important to obtain reproducible effects in animals, in terms of beneficial and pharmacokinetic results. For this reason, validated analytical methods for the quantification of the active compounds are necessary to guarantee product batches in compliance with what is stated in labels. The results demonstrated that the extraction method is highly reproducible, exhaustive, and efficient, with high recovery values for both the analytes at three different concentrations. Moreover, the developed analytical method has been shown to be precise, accurate, and adequate for the quantification of HES and CAR. Further studies will be carried out in order to evaluate the long-term stability of the feed additives in terms of degradation and/or loss of the active compounds. Furthermore, the nutraceutical effects of the final products will be investigated on livestock.

\section{Conflicts of Interest}

The authors declare no conflicts of interest regarding the publication of this paper. 


\section{References}

[1] Silbergeld, E.K. (2019) One Health and the Agricultural Transition in Food Animal Production. Global Transitions, 1, 83-92. https://doi.org/10.1016/j.glt.2019.01.003

[2] Elsasser, T.H., Klasing, K.C., Filipov, N. and Thompson, F. (2009) The Metabolic Consequences of Stress: Targets for Stress and Priorities of Nutrient Use. In: Moberg, G.P. and Mench, J.A., The Biology of Animal Stress: Basic Principles and Implications for Animal Welfare, Centre for Agriculture and Bioscience International, Wallingford, 77-110. https://doi.org/10.1079/9780851993591.0077

[3] Blokhuis, H.J., Hopster, H., Geverink, N.A., Korte, S.M. and Van Reenen, C.G. (1998) Studies of Stress in Farm Animals. Comparative Haematology International, 8, 94-101. https://doi.org/10.1007/BF02642498

[4] Falowo, A.B. and Akimoladun, O.F. (2020) Veterinary Drug Residues in Meat and Meat Products: Occurrence, Detection and Implications. In: Bekoe, S.O. and Saravanan, M., Eds., Veterinary Medicine and Pharmaceuticals, IntechOpen, London, 1-18. https://doi.org/10.5772/intechopen.83616

[5] European Food Safety Authority (2020) Report for 2018 on the Results from the Monitoring of Veterinary Medicinal Product Residues and Other Substances in Live Animals and Animal Products. EFSA Supporting Publications, 17, Article No. 1775E. https://doi.org/10.2903/sp.efsa.2020.EN-1775

[6] Sanz, D., Razquin, P., Condón, S., Juan, T., Juan, T., Herraiz, B., et al. (2015) Incidence of Antimicrobial Residues in Meat Using a Broad Spectrum Screening Strategy. European Journal of Nutrition \& Food Safety, 5, 156-165. https://doi.org/10.9734/EJNFS/2015/13795

[7] Rana, M.S., Lee, S.Y., Kang, H.J. and Hur, S.J. (2019) Reducing Veterinary Drug Residues in Animal Products: A Review. Food Science of Animal Resources, 39, 687-703. https://doi.org/10.5851/kosfa.2019.e65

[8] Kang, J.W., Park, H.C., Gedi, V., Park, S.J., Kim, M.A., Kim, M.K., et al. (2015) Veterinary Drug Residues in Domestic and Imported Foods of Animal Origin in the Republic of Korea. Food Additives and Contaminants. Part B Surveillance, 8, 106-112. https://doi.org/10.1080/19393210.2014.1001795

[9] Gallo, P., Fabbrocino, S., Dowling, G., Salini, M., Fiori, M., Perretta, G., et al. (2010) Confirmatory Analysis of Non-Steroidal Anti-Inflammatory Drugs in Bovine Milk by High-Performance Liquid Chromatography with Fluorescence Detection. Journal of Chromatography $A, 1217,2832-2839$.

https://doi.org/10.1016/j.chroma.2010.02.047

[10] Mehtabuddin, A.A., Ahmad, T., Nadeem, S., Tanveer, Z.I. and Arshad, J. (2012) Sulfonamide Residues Determination in Commercial Poultry Meat and Eggs. Undefined, 22, 473-478.

[11] Martin, G.B. and Ferasyi, T.R. (2016) Clean, Green, Ethical (CGE) Management: What Research Do We Really Need? The International Journal of Tropical Veterinary and Biomedical Research, 1, 1-8. https://doi.org/10.21157/ijtvbr.v1i1.5066

[12] European Parliament (2003) Regulation (EC) No 1831/2003 on the European Parliament and of the Council of 22 September 2003. On Additives for Use in Animal Nutrition. Official Journal of the European Communities, 268, 29-43.

[13] Surai, P.F. (2014) Polyphenol Compounds in the Chicken/Animal Diet: From the Past to the Future. Journal of Animal Physiology and Animal Nutrition, 98, 19-31. https://doi.org/10.1111/jpn.12070

[14] Tsiplakou, E., Pitino, R., Manuelian, C.L., Simoni, M., Mitsiopoulou, C., De Marchi, M., et al. (2021) Plant Feed Additives as Natural Alternatives to the Use of Synthetic 
Antioxidant Vitamins in Livestock Animal Products Yield, Quality, and Oxidative Status: A Review. Antioxidants, 10, Article No. 780. https://doi.org/10.3390/antiox10050780

[15] Stevanović, Z.D., Bošnjak-Neumüller, J., Pajić-Lijaković, I., Raj, J. and Vasiljević, M. (2018) Essential Oils as Feed Additives-Future Perspectives. Molecules, 23, Article No. 1717. https://doi.org/10.3390/molecules23071717

[16] Gadde, U., Kim, W.H., Oh, S.T. and Lillehoj, H.S. (2017) Alternatives to Antibiotics for Maximizing Growth Performance and Feed Efficiency in Poultry: A Review. Animal Health Research Reviews, 18, 26-45. https://doi.org/10.1017/S1466252316000207

[17] Hashemi, S.R. and Davoodi, H. (2011) Herbal Plants and Their Derivatives as Growth and Health Promoters in Animal Nutrition. Veterinary Research Communications, 35, 169-180. https://doi.org/10.1007/s11259-010-9458-2

[18] Elferink, E.V., Nonhebel, S. and Moll, H.C. (2008) Feeding Livestock Food Residue and the Consequences for the Environmental Impact of Meat. Journal of Cleaner Production, 16, 1227-1233. https://doi.org/10.1016/j.jclepro.2007.06.008

[19] European Parliament (2018) Regulation (EU) 2018/848 of the European Parl of 30 May 2018 on Organic Production and Labelling of Organic Products and Repealing Council Regulation (EC) No 834/2007. Official Journal of the European Communities, 150, 1-92.

[20] de la Rosa, J.D.P., Ruiz-Palomino, P., Arriola-Guevara, E., García-Fajardo, J., Sandoval, G. and Guatemala-Morales, G.M. (2018) A Green Process for the Extraction and Purification of Hesperidin from Mexican Lime Peel (Citrus aurantifolia Swingle) That Is Extendible to the Citrus Genus. Processes, 6, Article No. 266. https://doi.org/10.3390/pr6120266

[21] Di Mauro, A., Fallico, B., Passerini, A. and Maccarone, E. (2000) Waste Water from Citrus Processing as a Source of Hesperidin by Concentration on Styrene-Divinylbenzene Resin. Journal of Agricultural and Food Chemistry, 48, 2291-2295. https://doi.org/10.1021/jf990992w

[22] Russo, M., Bonaccorsi, I., Inferrera, V., Dugo, P. and Mondello, L. (2015) Underestimated Sources of Flavonoids, Limonoids and Dietary Fiber: Availability in Orange's By-Products. Journal of Functional Foods, 12, 150-157. https://doi.org/10.1016/j.jff.2014.11.008

[23] Tadros, F.J. and Andrade, J.M. (2021) Impact of Hesperidin in 100\% Orange Juice on Chronic Disease Biomarkers: A Narrative Systematic Review and Gap Analysis. Critical Reviews in Food Science and Nutrition. https://doi.org/10.1080/10408398.2021.1927976

[24] Kamboh, A.A., Memon, A.M., Mughal, M.J., Memon, J. and Bakhetgul, M. (2018) Dietary Effects of Soy and Citrus Flavonoid on Antioxidation and Microbial Quality of Meat in Broilers. Journal of Animal Physiology and Animal Nutrition, 102, 235-240. https://doi.org/10.1111/jpn.12683

[25] Hager-Theodorides, A.L., Massouras, T., Simitzis, P.E., Moschou, K., Zoidis, E., Sfakianaki, E., et al. (2021) Hesperidin and Naringin Improve Broiler Meat Fatty Acid Profile and Modulate the Expression of Genes Involved in Fatty Acid $\beta$-Oxidation and Antioxidant Defense in a Dose Dependent Manner. Foods, 10, Article No. 739. https://doi.org/10.3390/foods10040739

[26] Goliomytis, M., Kartsonas, N., Charismiadou, M., Symeon, G., Simitzis, P. and Deligeorgis, S. (2015) The Influence of Naringin or Hesperidin Dietary Supplementation on Broiler Meat Quality and Oxidative Stability. PLOS ONE, 10, Article ID: e0141652. https://doi.org/10.1371/journal.pone.0141652 
[27] Goliomytis, M., Simitzis, P., Papalexi, A., Veneti, N., Hager-Theodorides, A., Charismiadou, M., et al. (2019) Influence of Citrus Flavonoids on Laying Hen Performance, Inflammatory Immune Response, Egg Quality and Yolk Oxidative Stability. British Poultry Science, 60, 272-278. https://doi.org/10.1080/00071668.2019.1587150

[28] Fotakis, C., Lantzouraki, D., Goliomytis, M., Simitzis, P., Charismiadou, M., Deligeorgis, S., et al. (2017) NMR Metabolomics Investigates the Influence of Flavonoid-Enriched Rations on Chicken Plasma. Journal of AOAC International, 100, 315-322. https://doi.org/10.5740/jaoacint.16-0405

[29] Jugreet, B.S., Suroowan, S., Rengasamy, R.R.K. and Mahomoodally, M.F. (2020) Chemistry, Bioactivities, Mode of Action and Industrial Applications of Essential Oils. Trends in Food Science \& Technology, 101, 89-105.

https://doi.org/10.1016/j.tifs.2020.04.025

[30] Institut de Recerca i Tecnologia Agroalimentàries (2015) Review of Immune Stimulator Substances/Agents That Are Susceptible of Being Used as Feed Additives: Mode of Action and Identification of End-Points for Efficacy Assessment. EFSA Supporting Publications, 12, Article No. 905E. https://doi.org/10.2903/sp.efsa.2015.EN-905

[31] Castillo, M., Martín-Orúe, S.M., Roca, M., Manzanilla, E.G., Badiola, I., Perez, J.F., et al. (2006) The Response of Gastrointestinal Microbiota to Avilamycin, Butyrate, and Plant Extracts in Early-Weaned Pigs. Journal of Animal Science, 84, 2725-2734. https://doi.org/10.2527/jas.2004-556

[32] Veterinary Medicines Evaluation Unit (1997) VICH Topic GL2 (Validation: Methodology) Guideline on Validation of Analytical Procedures: Methodology Date for Coming into Operation by Vich gl2 (Validation Methodology).

[33] Mueller-Harvey, I. (2004) Modern Techniques for Feed Analysis. In: Assesing Quality and Safety of Animal Feeds, Food and Agriculture Organization of the United Nations, Rome, 1-38.

[34] Victor, M.M., David, J.M., Cortez, M.V.M., Leite, J.L. and da Silva, G.S.B. (2021) A High-Yield Process for Extraction of Hesperidin from Orange (Citrus sinensis L. osbeck) Peels Waste, and Its Transformation to Diosmetin, A Valuable and Bioactive Flavonoid. Waste and Biomass Valorization, 12, 313-320. https://doi.org/10.1007/s12649-020-00982-X

[35] Ma, Y., Ye, X., Hao, Y., Xu, G., Xu, G. and Liu, D. (2008) Ultrasound-Assisted Extraction of Hesperidin from Penggan (Citrus reticulata) Peel. Ultrasonics Sonochemistry, 15, 227-232. https://doi.org/10.1016/j.ultsonch.2007.03.006

[36] Pensel, P.E., Maggiore, M.A., Gende, L.B., Eguaras, M.J., Denegri, M.G. and Elissondo, M.C. (2014) Efficacy of Essential Oils of Thymus vulgaris and Origanum vulgare on Echinococcus granulosus. Interdisciplinary Perspectives on Infectious Diseases, 2014, Article ID: 693289. https://doi.org/10.1155/2014/693289 